\title{
The association of very low-density lipoprotein receptor (VLDLR) haplotypes with egg production indicates VLDLR is a candidate gene for modulating egg production
}

\author{
ZhePeng Wang, GuoHua Meng, Na Li, MingFen Yu, XiaoWei Liang, YuNa Min, FuZhu Liu and \\ YuPeng Gao \\ College of Animal Science and Technology, Northwest A\&F University, Yangling, Shaanxi, China.
}

\begin{abstract}
The very low-density lipoprotein receptor (VLDLR) transports egg yolk precursors into oocytes. However, our knowledge of the distribution patterns of VLDLR variants among breeds and their relationship to egg production is still incomplete. In this study, eight single nucleotide polymorphisms (SNPs) that account for $87 \%$ of all VLDLR variants were genotyped in Nick Chick (NC, $n=91)$, Lohmann Brown (LohB, $n=50)$ and Lueyang ( $L Y, n=381)$ chickens, the latter being an Chinese indigenous breed. Egg production by NC and LY chickens was recorded from 17 to 50 weeks. Only four similar haplotypes were found in NC and LohB, of which two accounted for $100 \%$ of all NC haplotypes and 92.5\% of LohB haplotypes. In contrast, there was considerable haplotypic diversity in LY. Comparison of egg production in LY showed that hens with NC-like haplotypes had a significantly higher production $(p<0.05)$ than those without the haplotypes. However, VLDLR expression was not significantly different between the haplotypes. These findings indicate a divergence in the distribution of VLDLR haplotypes between selected and non-selected breeds and suggest that the near fixation of VLDLR variants in NC and LohB is compatible with signature of selection. These data also support VLDLR as a candidate gene for modulating egg production.
\end{abstract}

Keywords: chicken, egg production, haplotype, very low-density lipoprotein receptor (VLDLR).

Received: August 13, 2015; Accepted: February 20, 2016.

\section{Introduction}

In the poultry industry, egg production is an important trait that determines economic profits. Therefore, understanding the genetic factors that govern this trait is of great significance to geneticists, breeders and producers. However, because of the complex genetic factors and possible environmental influences that can interfere with egg production, our knowledge of the molecular regulatory mechanisms underlying this trait is still limited.

Egg production involves the development of oocytes and ovulation. In this process, the oocytes will take up precursors for yolk formation from the circulation, grow from 6-7 $\mathrm{mm}$ to $35 \mathrm{~mm}$ and finally undergo ovulation (Bujo et al., 1997). Very low-density lipoprotein (VLDL) and vitellogenin (VTG), two key yolk precursors that transport lipids and account for more than $30 \%$ of yolk weight, are taken up by oocytes via receptor-mediated endocytosis (Bujo et al., 1997).

The very low-density lipoprotein receptor (VLDLR) is the key carrier of VLDL and VTG (Bujo et al., 1994),

Send correspondence to Yupeng Gao. College of Animal Science and Technology, Northwest A\&F University, Xinong Road No. 22, Yangling, $\quad 712100, \quad$ Shaanxi, China. E-mail: gaoyupeng112@sina.com which suggests that changes in the expression and function of the VLDLR gene may influence oocyte development and subsequent egg production. This inference is supported by a study showing that hens with a non-synonymous mutation involving C682S (defined as the RO mutation) in the VLDLR fail to lay eggs and display sever hyperlipidemia (Bujo et al., 1995). The level of ovarian VLDLR expression is also correlated with the rejuvenation of reproductive performance in molted hens (Meng et al., 2013) and with egg mass, clutch size and laying interval in zebra finch (Han et al., 2009).

With regard to the mapping of quantitative trait loci (QTL), several genome-wide association and selective sweep analyses have attempted to identify genomic regions associated with egg production and quality traits by using high-density single nucleotide polymorphism (SNP) chips or whole-genome sequencing (Rubin et al., 2010; Liu et al., 2011; Elferink et al., 2012; Wolc et al., 2012, 2014; Gholami et al., 2015; Sun et al., 2015a,b; Yi et al., 2015; Yuan et al., 2015). However, because of lower genetic variation on chromosome Z (Sundström et al., 2004) and the limitations of statistical methods, the $\mathrm{Z}$ chromosome has been consistently excluded from most studies (Rubin et al., 2010; Elferink et al., 2012; Gholami et al., 2015; Sun et al., 
2015a,b; Yi et al., 2015; Yuan et al., 2015), or limited Z-linked SNPs have been used (Liu et al., 2011; Wolc et al., 2012, 2014). This means that the Z-linked VLDLR, despite being a functionally important candidate, has not been identified by high-throughput mapping studies. Nevertheless, the results of some low-accuracy mappings have suggested that the VLDLR is a promising candidate gene for modulating egg production. Specifically, several VLDLR variants show a significant association with egg weight, age at first egg and egg production in chickens, ducks and quails (Wang et al., 2011; Cao et al., 2012; Wu et al., 2015). QTL intervals for egg number and age at first egg cover the VLDLR (Tuiskula-Haavisto et al., 2002; Sasaki et al., 2004; Xu et al., 2011) and the mapping intervals for Haugh units, egg weight, yolk height and egg production rate locate near the VLDLR (Atzmon et al., 2007; Honkatukia et al., 2013; Goto et al., 2014).

Despite the foregoing studies, there is still only limited information on the distribution patterns of VLDLR variants among chicken breeds and the relationship between these variants and egg production. The aim of this work was therefore to examine the sequence variants of VLDLR among Nick Chick (NC), Lohmann Brown (LohB) and Lueyang (LY) chickens, the latter being a Chinese indigenous breed. The relationship between VLDLR haplotypes, VLDLR mRNA expression and egg production was also analyzed.

\section{Material and Methods}

\section{Birds and data collection}

Three breeds of chickens (NC, LohB and LY) were used in this study. NC and LohB represent commercial white- and brown-egg layer breeds, respectively, specifically selected for a spectrum of egg production and quality traits. LY is a domesticated unselected indigenous breed from Lueyang town in Shaanxi province, China. Historically, LY chickens have not been specifically selected for production traits. Consequently, the breed shows very poor egg production performance and large intraspecies variability, with an average of 85-115 eggs per year.

Fertilized NC eggs were collected from 135 half-sib families whereas fertilized LY eggs were from a free-range, random-mating population of approximately 1,800 birds with male:female ratio of 1:20. Two experimental populations of $500 \mathrm{NC}$ ( 450 hens and 50 roosters) and 2,853 LY (1,543 hens and 1,310 roosters) born in the same hatch were reared on the experimental station at Northwest A\&F University from December, 2012 to November, 2013. These birds were reared in single-hen cages with feed and water ad libitum. Egg production of $100 \mathrm{NC}$ and $500 \mathrm{LY}$ that were randomly selected from the above experimental populations was recorded from 17 to 50 weeks of age. Finally, $91 \mathrm{NC}$ and $381 \mathrm{LY}$ with complete egg production records were used in the subsequent association study. Fifty LohB were randomly sampled from a commercial population of approximate 20,000 birds.

\section{Estimation of egg production parameters}

The weekly number of eggs per bird was recorded from 17 to 50 weeks of age and the individual egg production rate was calculated as the weekly number of egg divided by seven days. Based on the data, several mean population egg production parameters were estimated using the three nonlinear regression models indicated below:

1. Segmented polynomial model (Fialho and Ledur, 1997):

$$
y=\left\{\begin{array}{c}
p-3 \times p \times\left(\frac{t p-t}{t i p}\right)^{2}+2 \times p \times\left(\frac{t p-t}{t i p}\right)^{3}, t p-t i p \leq t \leq t p \\
p-s(t-t p), \quad t p \leq t,
\end{array}\right.
$$

where $\mathbf{p}$ is the peak egg production rate, $\mathbf{s}$ is the weekly decrease in egg production rate after the peak, tp is the age of the hen at the peak and tip is the time interval between the start and peak of egg production.

2. Yang model (Yang et al., 1989):

$$
y=\frac{\mathrm{a} e^{-\mathrm{xt}}}{1+e^{-\mathrm{c}(\mathrm{t}-\mathrm{d})}}
$$

where $\mathbf{a}$ is a scale parameter, $\mathbf{c}$ is a reciprocal indicator of the variation in sexual maturity, $\mathbf{x}$ is the weekly decrease in egg production rate after the peak production and $\mathbf{d}$ is the mean age of sexual maturity of the hens.

$$
\text { 3. Persistency model (Grossman et al., 2000): }
$$

$$
\begin{aligned}
& y=0.3 \times\left(\frac{y p}{t 2-t 1}\right) \times\left[\ln \left(\frac{e^{\frac{t}{0.3}}+e^{\frac{t 1}{0.3}}}{1+e^{\frac{t 1}{0.3}}}\right)-\ln \left(\frac{e^{\frac{t}{0.3}}+e^{\frac{t 2}{0.3}}}{1+e^{\frac{t 2}{r 0.3}}}\right)\right]+ \\
& 0.3 \times b 4 \times \ln \left(\frac{e^{\frac{t}{0.3}}+e^{\frac{(t 2+P)}{0.3}}}{1+e^{\frac{(t 2+P)}{0.3}}}\right)
\end{aligned}
$$

where yp is the egg production rate at the peak production, $\mathbf{t} \mathbf{1}$ is the time at the transition from a slow increase to a rapid increase in the egg production rate, $\mathbf{t} \mathbf{2}$ is the time at the transition from a rapid increase to a constant rate of egg production, $\mathbf{P}$ is the duration of the period of constant production, and $\mathbf{b 4}$ is the weekly decrease in egg production rate after constant production.

All parameters were estimated using the nonlinear least-square method by searching for the set of parameters that produced the smallest sum of the squared errors. The nonlinear least-squares estimate was obtained by using the Gauss-Newton algorithm in the NLIN procedure of SAS v9.2 software with a default convergence criterion of $10^{-5}$ (SAS Institute Inc., 2008). 


\section{Evaluation of the goodness of fit of the three nonlinear models}

Four statistics, namely, Akaike's information criterion (AIC), mean square error (MSE), coefficient of determination $\left(\mathrm{R}^{2}\right.$ ) and mean model error (MME), were used to evaluate the goodness of fit of the three nonlinear models.

\section{$A / C$}

The AIC is a statistic based on information theory. In contrast to $\mathrm{R}^{2}$ (see below), AIC cannot evaluate the quality of a model in an absolute sense. Rather, given a set of candidate models, AIC can provide a relative estimate of the quality of these models, with the preferred model being the one with the minimum AIC value. AIC is calculated as follows:

$\mathrm{AIC}=\mathrm{n} \times \operatorname{Ln}\left(\mathrm{SS}_{\text {error }} / \mathrm{n}\right)+2 \times \mathrm{k}($ Savegnago et al.

where $\mathbf{n}$ is the number of records used for parameter estimation, $\mathbf{S S}_{\text {error }}$ is the sum of the squared error of the model, and $\mathbf{k}$ is the number of parameters in the model.

MSE

MSE is calculated as follows:

$$
\mathrm{MSE}=\frac{\sum_{i=1}^{n} \sum_{t=1}^{m}\left(y_{i t}-\hat{y}_{i t}\right)^{2}}{n m-p}
$$

where $\mathbf{y}_{\mathrm{it}}$ is the observed weekly egg production rate of hen $i$ at week $t, \hat{\mathbf{y}}_{\text {it }}$ is the predicted weekly egg production rate of hen $i$ at week $t, \mathbf{n}$ is the number of hens, $\mathbf{m}$ is the number of weeks during which egg production was recorded, and $\mathbf{p}$ is the number of parameters in the model.

$R^{2}$

$\mathrm{R}^{2}$ is calculated as follows:

$\mathrm{R}^{2}=\frac{\mathrm{SS}_{\text {model }}}{\mathrm{SS}_{\text {total }}}$

where $\mathrm{SS}_{\text {model }}$ is the sum of the squares of the model and $\mathrm{SS}_{\text {total }}$ is the total sum of the squares.

\section{MME}

MME is the mean of all model errors. One advantage of MME is that the statistic not only evaluates the goodness of fit of a model, but also reflects the direction in which observed values deviate from predicted values (Savegnago et $a l ., 2012)$. A positive MME means that the model overestimates the egg production rate as a whole; correspondingly, a negative MME indicates that the egg production rate is underestimated. MME is calculated as follows:

$$
\mathrm{MME}=\frac{\sum_{t}^{m} \frac{\left(\hat{y}_{t}-\bar{y}_{t}\right)}{\bar{y} t}}{m}
$$

where $\hat{y}_{t}$ is the average predicted egg production rate at week $t, \bar{y}_{t}$ is the average observed egg production rate at week $t$, and $\mathbf{m}$ is the number of weeks during which egg production was recorded. In this study, $\mathbf{m}$ was equal to 34 (from 17 to 50 weeks).

The flexibility of the models and the differences in nonlinear trends among groups were assessed by plotting and analyzing the fitted egg production curves and the average observed weekly egg production rates.

\section{Comparison of egg production parameters among different groups}

Differences in the egg production parameters between LY and NC and among four haplotype groups were compared statistically using the sum of squares reduction test (SSRT; SAS Institute Inc., 2008). The null hypothesis for the SSRT, also known as a reduced model, is that there is no significant difference $(p>0.05)$ in the nonlinear regression trends between groups such that the same set of parameters should exist across groups. In contrast, the alternative hypothesis, corresponding to a full model, assumes that nonlinear trends should vary across groups and that different sets of parameters are required to fit the egg production rates of different groups. The main idea of SSRT is to statistically indicate whether the full model, which applies more sets of parameters, provides a significantly better fit than the reduced model. An $\mathrm{F}_{\mathrm{R}}$ statistic was calculated as:

$$
\mathrm{FR}=\frac{\left(\mathrm{SSE}_{\mathrm{r}}-\mathrm{SSE}_{\mathrm{f}}\right) /\left(\mathrm{df}_{\mathrm{r}}-\mathrm{df}_{\mathrm{f}}\right)}{\mathrm{SSE}_{\mathrm{f}} / \mathrm{df}_{\mathrm{f}}}
$$

where $\mathrm{SSE}_{\mathrm{r}}$ and $\mathrm{SSE}_{\mathrm{f}}$ indicate the residual sum of squares of the reduced model and full model, respectively, with $\mathrm{df}_{\mathrm{r}}$ and $\mathrm{df}_{\mathrm{f}}$ being the error degrees of freedom. Because the difference $\mathrm{df}_{\mathrm{r}}-\mathrm{df}_{\mathrm{f}}$ corresponds to the number of increased parameters in the full model, the numerator of the $F_{R}$ statistic can be explained as the mean contribution that every additional parameter in the full model makes to the reduction in the residual sum of squares. After scaling the full model by the mean squared error, the contribution is statistically evaluated by comparing the $F_{R}$ statistic and quantiles from an $\mathrm{F}$ distribution with the $\left(\mathrm{df}_{\mathrm{r}}-\mathrm{df}_{\mathrm{f}}\right)$ numerator and $\mathrm{df}_{\mathrm{f}} \mathrm{de}-$ nominator degrees of freedom.

In the event of the null hypothesis being rejected, it is necessary to elucidate whether all parameters, or only some of them, varied across groups. For this, parameter differences were examined using the method mentioned in Example 60.5 of the SAS/STAT ${ }^{\circledR} 9.22$ User's Guide (SAS Institute Inc., 2008). Briefly, parameter differences ( $\Delta$ par) between two groups and the $95 \%$ confidence interval of $\Delta$ par were estimated by a simple reparameterization in 
which Par2 (parameters of group 2) were replaced by Par1 (parameters of group 1) $+\Delta$ par. The statistical significance of $\Delta$ par would be confirmed if the $95 \%$ confidence interval of $\Delta$ par excluded 0 (SAS Institute Inc., 2008). The SSRT was run using SAS 9.2 software (SAS Institute Inc., 2008).

\section{Analysis of VLDLR genomic sequence variants}

Blood was collected from a wing vein into anticoagulant (ACD - acid citrate dextrose solution) and immediately stored at $-20{ }^{\circ} \mathrm{C}$. Genomic DNA was extracted from blood using the phenol/chloroform method (Sambrook and Russell, 2006). A genomic interval of chromosome $\mathrm{Z}$ (chrZ; 26411455-26431662) covering the whole VLDLR gene and a $5 \mathrm{~kb}$ upstream region was re-sequenced using 10 LY chickens randomly selected from the 381 specimens mentioned above. The primers used for polymerase chain reaction (PCR) amplification and Sanger sequencing are listed in Table S1. VLDLR sequence variants were found by sequence alignment using ChromasPro 1.5.

SNP genotypes were detected by PCR-restriction fragment length polymorphism (PCR-RFLP). The primers and restriction enzymes used in PCR-RFLP are shown in Table 1. Part of the PCR-RFLP results were also verified by Sanger sequencing.

\section{Detection of the RO mutation in LY chickens}

Fifty LY hens with an egg number $<100$ from 17 to 50 weeks and $30 \mathrm{LY}$ roosters were selected from the 381 LY hens and 1,310 roosters. The RO mutation was identified using PCR-RFLP. A $559 \mathrm{bp}$ fragment containing the RO locus was obtained by PCR using the forward primer 5'-TCTATGGTGCCAACAAAT-3' and the reverse primer 5'-CATCTCAGACCGTCCTCC-3'. After digestion of the PCR products with Eco57I (Life Technologies, Shanhai, China) at $37{ }^{\circ} \mathrm{C}$ for $2 \mathrm{~h}$, the products were separated on a $2 \%$ agarose gel. Since the 559 bp fragment contained two Eco57I cleavage sites at $85 \mathrm{bp}$ and $451 \mathrm{bp}$, wild-type birds should show two bands ( 85 bp and $474 \mathrm{bp}$ ), whereas those with the RO mutation should have four bands (85 bp, $474 \mathrm{bp}, 108 \mathrm{bp}$ and $451 \mathrm{bp}$ ).

\section{Detection of VLDLR mRNA expression in ovary and liver}

The levels of VLDLR mRNA expression in ovary and liver were compared among $\mathrm{NC}(\mathrm{n}=6)$ and $\mathrm{LY}$ chickens with $(n=6)$ and without $(n=6)$ the $\operatorname{ATAATA}(\mathrm{A} / \mathrm{C}) \mathrm{T}$ haplotypes. All birds were 34 weeks old and were selected from the LY and NC populations described above. The birds were rapidly killed after being anaesthetized with 5 $\mathrm{mg} / \mathrm{kg}$ of Zoletil by intramuscular injection. Liver and ovary with previtellogenic follicles (diameter $<5 \mathrm{~mm}$ ) were immediately removed and immersed in RNAlater solution (CWBio Corp., Beijing, China) at $4{ }^{\circ} \mathrm{C}$ overnight and then stored at $-80^{\circ} \mathrm{C}$ until used. The use and care of birds in

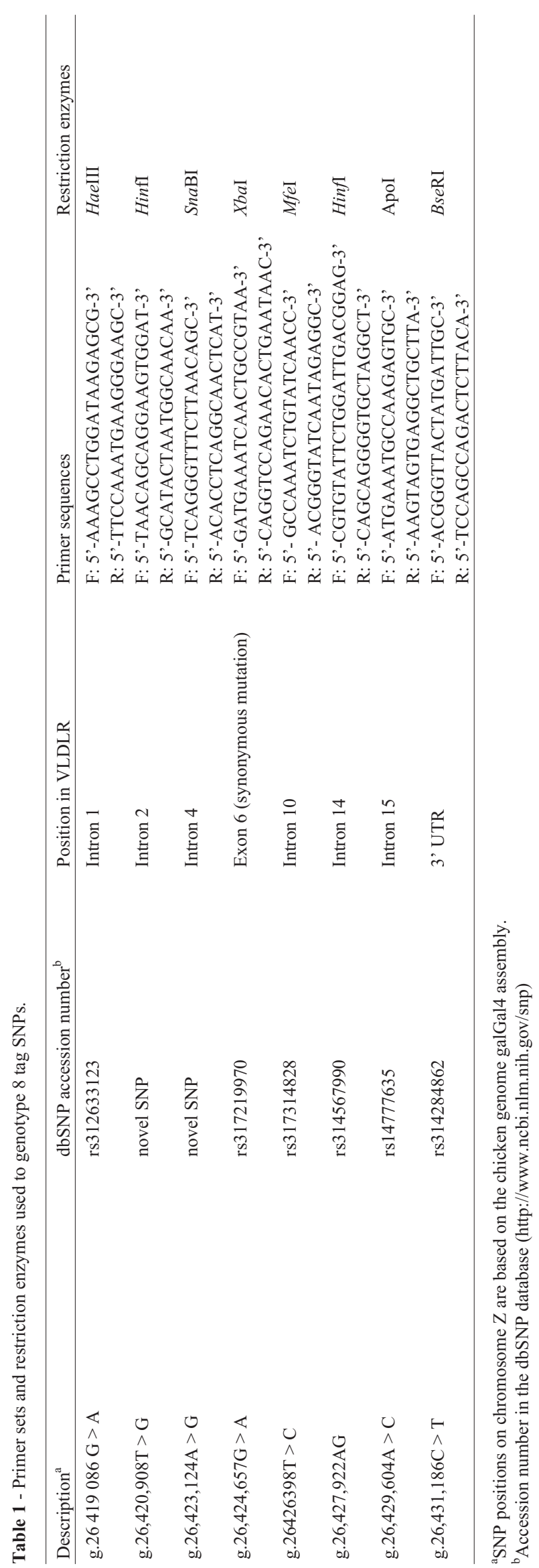


this study was approved by the Northwest A\&F University Ethics Committee.

Total RNA was extracted from tissues using TRIzol (CWBio Corp.), according to the manufacturer's instructions. The quality (intactness) of the RNA was assessed visually after electrophoresis in 1\% agarose gels and further confirmed by an RNA integrity number (RIN) [\#GTEQ\#] 7 provided by BioAnalyzer 2100 (Agilent Technologies Corp., Santa Clara, CA, USA). One microgram of total RNA was transcribed to cDNA with a HiFi MMLV first-strand cDNA synthesis kit (CWBio Corp.), according to the manufacturer's instructions. Briefly, RNA was added to $20 \mu \mathrm{L}$ of reaction mixture that consisted of $4 \mu \mathrm{L}$ of dNTP $\operatorname{mix}(2.5 \mathrm{mM}$ each), $2 \mu \mathrm{L}$ of oligo dT primer $(20 \mu \mathrm{M}), 4 \mu \mathrm{L}$ of 5RT buffer ( $250 \mathrm{mM}$ Tris-HCl, $\mathrm{pH} 7.6,375 \mathrm{mM} \mathrm{KCl}, 15$ $\mathrm{mM} \mathrm{MgCl} 2), 2 \mu \mathrm{L}$ of $0.1 \mathrm{M}$ DTT, $1 \mu \mathrm{L}$ of $200 \mathrm{U}$ of $\mathrm{HiFi}-\mathrm{MMLV} / \mu \mathrm{L}$ and RNase-free water. The reaction conditions were $42{ }^{\circ} \mathrm{C}$ for $45 \mathrm{~min}$ for cDNA synthesis and then $85^{\circ} \mathrm{C}$ for $5 \mathrm{~min}$ to inactivate MMLV reverse transcriptase.

The expression of VLDLR in ovary and liver was detected using quantitative real-time PCR (qPCR). The reactions were run using an UltraSYBR mixture qPCR kit (CWBio Corp.) in a total volume of $20 \mu \mathrm{L}$ containing $1 \mu \mathrm{L}$ of cDNA, $0.3 \mu \mathrm{L}$ of primer pairs ( $4 \mu \mathrm{M}$ each), $10 \mu \mathrm{L}$ of 2 UltraSYBR mixture and $8.4 \mu \mathrm{L}$ of RNase-free water. The reactions were run in an iQ5 real-time PCR detection platform (Bio-Rad Laboratories, Inc, Hercules, CA, USA) using the cycling conditions described in the UltraSYBR mixture protocol sheet. Three technical replicates were run for every sample. The resulting $\mathrm{Ct}$ data were analyzed using the $2^{-} \Delta \Delta \mathrm{CT}$ method (Livak and Schmittgen, 2001). GAPDH was used as an internal reference (housekeeping gene) to normalize the amount of cDNA input. Samples from birds without the ATAATA(A/C)T haplotypes were used as calibrators and the level of VLDLR expression in birds with ATAATA(A/C)T haplotypes and in NC chickens was expressed as the fold-change relative to the calibrator. The forward and reverse primers were 5'-TGTGGTCCTCAGTCAACC-3' and 5'-TCTGCTGCACTACAAGTCA-3' for VLDLR and 5'-ATACACAGAGGACCAGGTTG-3', and 5'-AAACTCATTGTCATACCAGG-3' for GAPDH.

\section{Results}

\section{Description of the goodness of fit of the three nonlinear models}

Egg production records from the LY, NC and four LY haplotype subgroups were fitted using the Segmented Polynomial, Yang and Persistency models. The quality of fit of the models was assessed statistically (Table 2) and graphically (Figures 1 and 2). The three models showed similar goodness of fit when dealing with the data from the LY or NC, which was supported by the almost identical AIC, MSE and $\mathrm{R}^{2}$ values (Table 2). Exceptionally, the MME value showed considerable variation among the three models. For the NC group, the MME statistic suggested that the

Table 2 - Statistical criteria used to evaluate the goodness of fit of three nonlinear models in fitting the data from Lueyang and Nick Chick chickens ${ }^{\mathrm{a}}$

\begin{tabular}{llllll}
\hline Groups & Models & AIC & MSE & $\mathrm{R}^{2}$ & MME \\
\hline NC & Segmented polynomial & -6491.4 & 0.0219 & 0.968 & -0.00124 \\
& Yang & -6493.9 & 0.0219 & 0.967 & 0.0393 \\
LY & Persistency & -6489.6 & 0.0219 & 0.968 & -0.0116 \\
& Segmented polynomial & -22892.2 & 0.0748 & 0.663 & -0.119 \\
& Yang & -22906.5 & 0.0747 & 0.663 & -0.110 \\
ATAATA(A/C)T & Persistency & -22893.2 & 0.0748 & 0.663 & -0.127 \\
& Segmented polynomial & -2674.7 & 0.0646 & 0.758 & -0.114 \\
& Yang & -2678.9 & 0.0644 & 0.759 & -0.0965 \\
GGAACACT & Persistency & -2672.4 & 0.0647 & 0.758 & -0.116 \\
& Segmented polynomial & -5356.7 & 0.0735 & 0.684 & -0.104 \\
GGGGCGCC & Yang & -5356.7 & 0.0734 & 0.684 & -0.0716 \\
& Persistency & -5354.7 & 0.0734 & 0.684 & -0.100 \\
& Segmented polynomial & -6283.3 & 0.0747 & 0.649 & -0.115 \\
& Yang & -6285.9 & -6281.3 & 0.0746 & -0.101 \\
& Persistency & -4361.6 & 0.0746 & 0.649 & -0.123 \\
& Segmented polynomial & -4366.9 & 0.0761 & 0.629 & -0.102 \\
& Yang & -4359.6 & 0.0759 & 0.629 & -0.0958 \\
& Persistency & & 0.0761 & -0.101 \\
\hline
\end{tabular}

${ }^{a} \mathrm{AIC}$ - Akaike's information criterion, MSE - mean square error, $\mathrm{R}^{2}$ - coefficient of determination, MME - mean model error. 

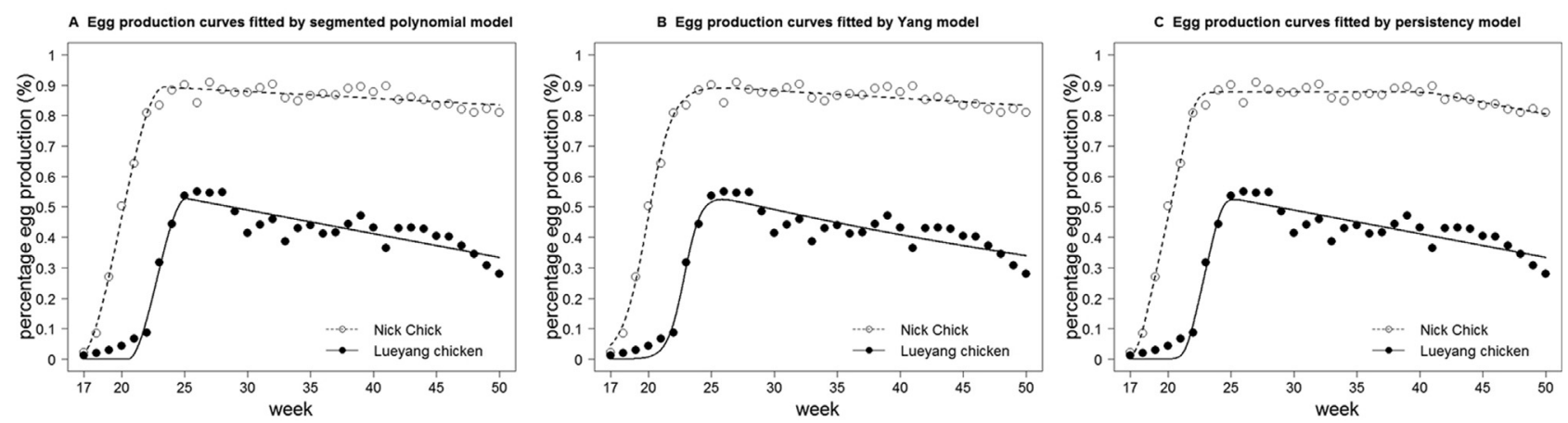

Figure 1 - Average weekly egg production rate and fitted egg production curves for Lueyang and Nick Chick chickens.
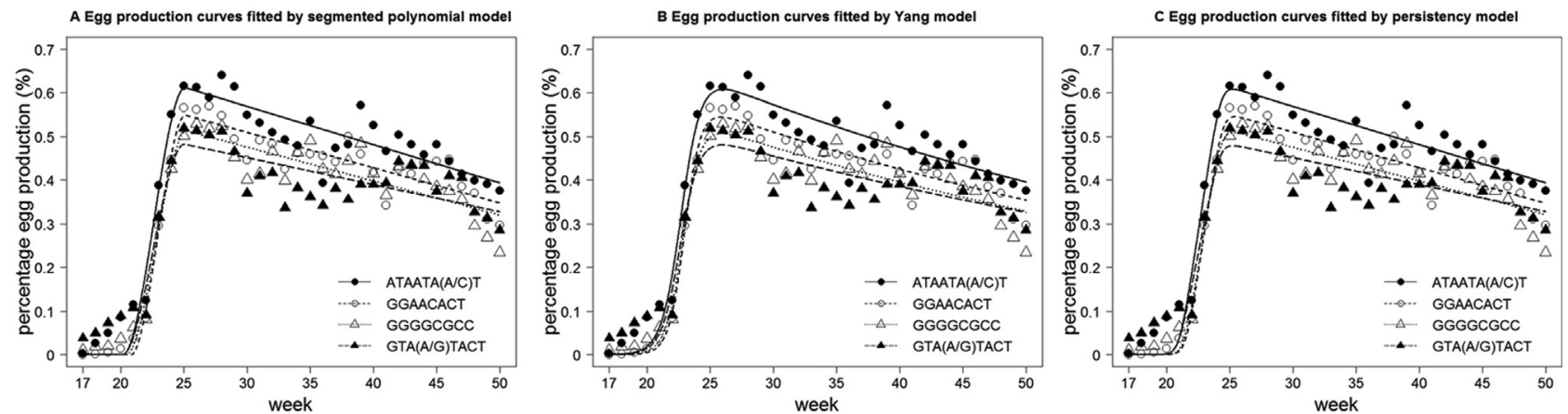

Figure 2 - Average weekly egg production rates and the fitted egg production curves for different LY haplotypes. ATAATA(A/C)T represented two haplotypes (ATAATAAT and ATAATACT), as did GTA(A/G)TACT.

Segmented Polynomial model provided the best fit, whereas for the LY and four LY haplotype subgroups the MME values were consistently close to zero in the Yang model, which suggested that this was the best model (Table 2). Compared to the weak effect of the models per se on the goodness of fit, the data themselves exerted a more important role in determining the goodness of fit. Thus, higher $\mathrm{R}^{2}$ and lower MSE and MME indicated that the goodness of fit of the three models was better for NC data than for LY (Table 2).

By plotting the fitted curve and the average observed weekly egg production rates, it was possible to graphically assess the flexibility of the models. In line with results from the statistical evaluation, all three models displayed excellent flexibility in adjusting to changes in the egg production rate of NC. However, deviations of the fitted curves from the data for LY were severe, especially for the egg production rates from 17 to 21 weeks, for which these models did not show enough flexibility to slow the increase in egg production rates (Figure 1).

\section{Comparison of egg production between LY and NC chickens}

By using the three nonlinear models, 13 egg production parameters were estimated and used to evaluate egg production performance in LY and NC. These parameters were classified into two categories: category 1 evaluated the changes in egg production rate, such as peak production rate (parameters $\mathbf{P}$ and $\mathbf{y p}$ ) and the decrease in egg production rate after the peak ( $\mathbf{s}, \mathbf{x}$ and $\mathbf{b 4}$ ); category 2 evaluated the changes in egg production time, such as age at the first egg ((tp-tip), t2), time to reach the production peak (tp and t2), sexual maturity (d) and the maintenance of constant production (persistency) (Table 2). LY chickens had a lower production peak (0.53 vs.0.89 for $\mathbf{P})$, larger decrease in the egg production rate $(0.0078 v s .0 .0022$ for $\mathbf{s})$, longer time until the start of egg laying (21.4 vs. 17.5 for t1), peak production (25.1 vs. 23.3 for tp) and sexual maturity (22.9 vs. 19.9 for $\mathbf{d}$ ), as well as zero persistency (0 vs. 18.5), when compared to NC (Table 3, Figure 1). The differences in these parameters indicated that egg production was lower in LY than in NC.

\section{Distribution of VLDLR variants among NC, LohB and LY chickens}

Re-sequencing identified 30 SNPs in the VLDLR (Table S2). Of these SNPs, eight accounted for $87 \%$ of all variants and were selected as tag SNPs and finally genotyped in LY $(n=381), N C(n=91)$ and LohB $(n=50)$ chickens. VLDLR variants were almost fixed in NC and LohB, with two main haploytpes accounting for $100 \%$ of all NC haplotypes and $92.5 \%$ of LohB haplotypes (Table 4). In contrast, high sequence polymorphism was observed in LY chickens, for which 14 haplotypes were found (Table 4). 


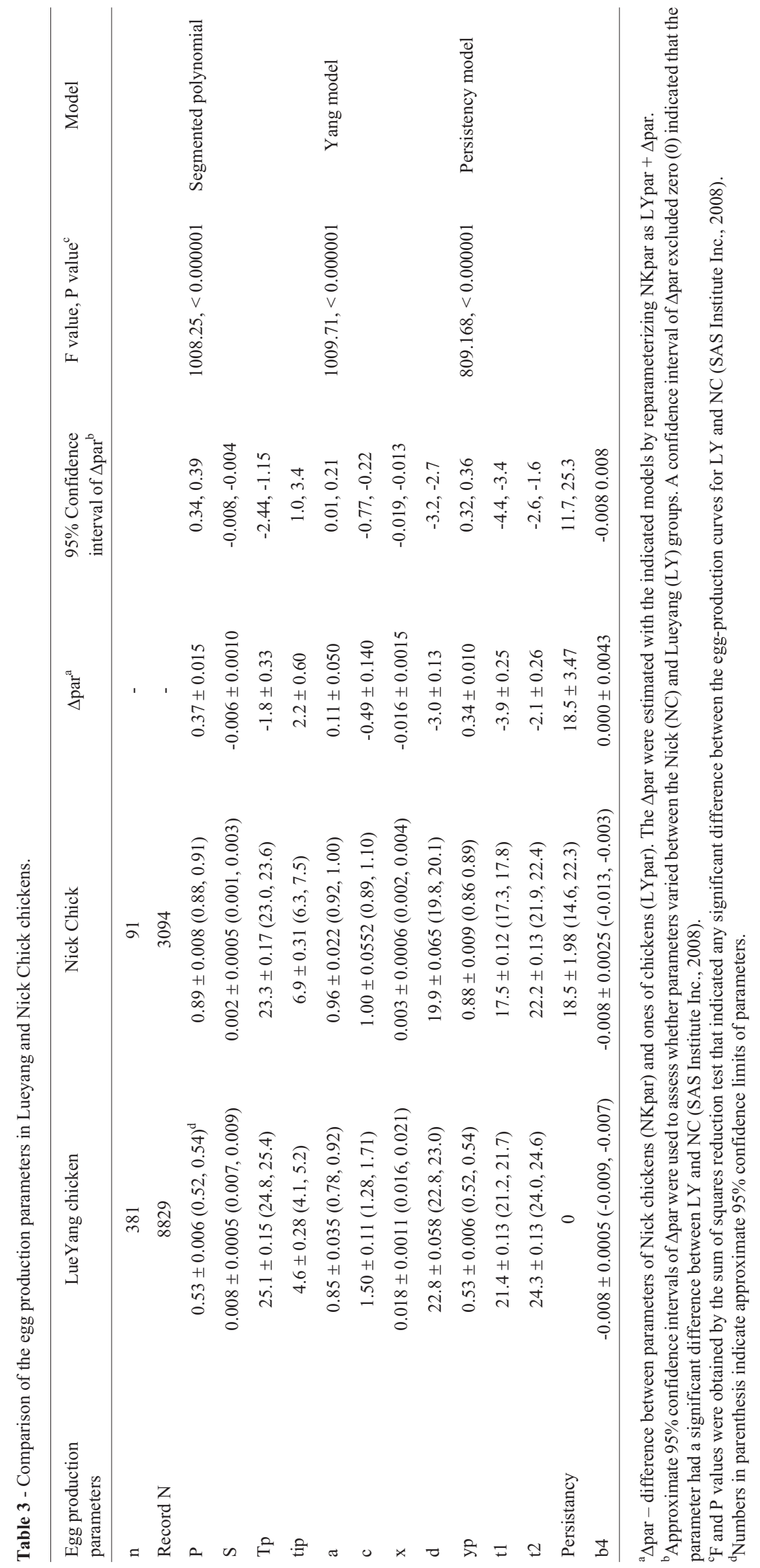


Table 4 - VLDLR haplotype frequency distributions in Nick Chick, Lohmann Brown and Lueyang chickens.

\begin{tabular}{lcccc}
\hline Name & Haplotype & $\begin{array}{c}\text { Lueyang } \\
(\mathrm{n}=381)\end{array}$ & $\begin{array}{c}\text { Nick Chick } \\
(\mathrm{n}=91)\end{array}$ & $\begin{array}{c}\text { Lohmann } \\
(\mathrm{n}=50)\end{array}$ \\
\hline LYhap1 & GGGGCGCC & 0.283 & - & - \\
LYhap2 & GGAACACT & 0.228 & - & - \\
LYhap3 & GTAGTACT & 0.108 & - & - \\
LYhap4 & GTAATACT & 0.089 & - & - \\
LYhap5 & ATAATAAT & 0.079 & - & - \\
LYhap6 & GGGACGCC & 0.042 & - & - \\
LYhap7 & GGGGCACT & 0.024 & - & - \\
LYhap8 & ATAATACT & 0.032 & - & - \\
LYhap9 & GGGGCGCT & 0.018 & - & - \\
LYhap10 & GGGACGCT & 0.021 & - & - \\
LYhap11 & GTAGTAAT & 0.018 & - & - \\
LYhap12 & GTAGTACC & 0.018 & - & - \\
LYhap13 & GGAGCGCC & 0.018 & - & - \\
LYhap14 & GGAACGCC & 0.021 & - & - \\
NChap1 & ATAATAAC & - & 0.66 & - \\
NChap2 & ATAACAAC & - & 0.34 & 0.925 \\
Lohhap1 & ATAACACC & - & - & 0.05 \\
Lohhap2 & ATGACACC & - & - & 0.025 \\
\hline
\end{tabular}

${ }^{a}$ The haplotypes in bold letters were the two LY haplotypes that showed the highest similarity to two NC haplotypes.

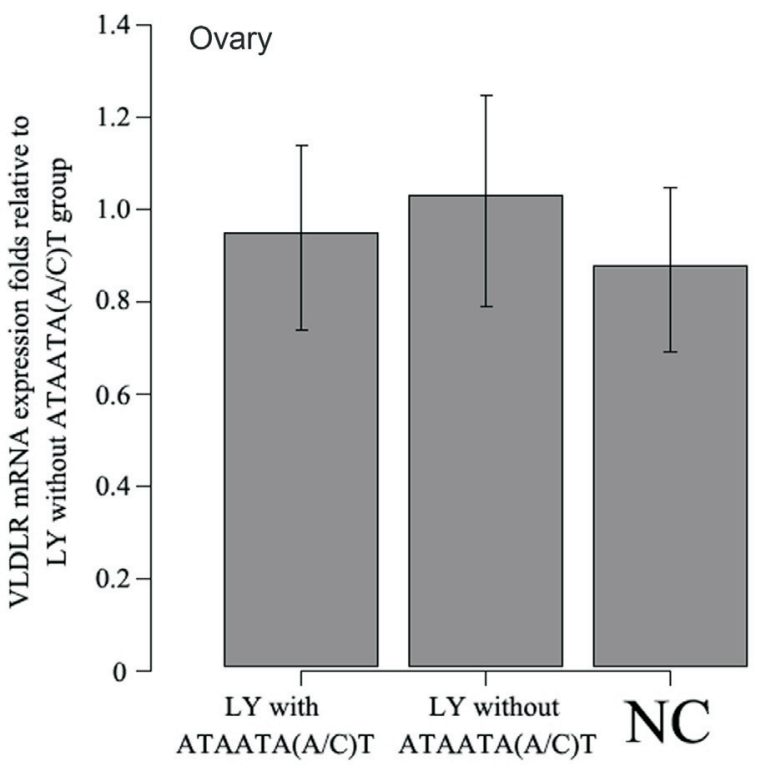

\section{Comparison of egg production among different VLDLR haplotypes}

Of 14 haplotypes found in LY, two (ATAATAAT, ATAATACT) showed high similarity to two NC haplotypes (ATAATAAC, ATAACAAC) and are referred to below as NC-like haplotypes. Egg production was compared between the two NC-like haplotypes and the other LY haplotypes. After discarding haplotypes with frequencies $<5 \%$ and combining highly similar haplotypes, we classified the egg production records from $312 \mathrm{LY}$ into four haplotype groups, and then the 13 parameters indicated above were estimated for each group (Table 5). A single significant difference was observed in the peak production rate (Table 5, Figure 2 ). The differences ( $\Delta$ par) observed in the remaining parameters were not significant since the $95 \%$ confidence interval of $\Delta$ par contained zero (0) (Table 5).

\section{Haplotype-specific expression analysis of VLDLR}

The level of VLDLR mRNA expression was compared among the LY and NC-like haplotypes $(n=6)$, LY without the haplotypes $(\mathrm{n}=6)$ and $\mathrm{NC}(\mathrm{n}=6)$. There was no significant difference in the expression of VLDLR mRNA in ovary and liver between any two groups (Figure 3).

\section{Detection of the RO mutation in LY chickens}

The RO mutation is a missense mutation that leads to a failure to lay eggs and results in hyperlipidemia (Bujo et al., 1995). Although there is no change in reproductive function, roosters with this mutation transmit it to one-half

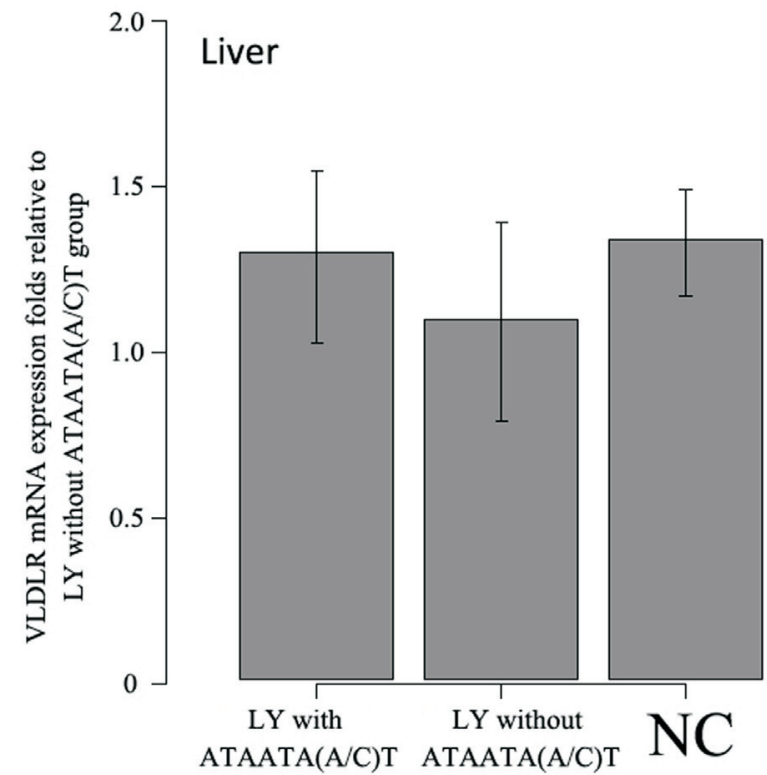

Figure 3 - Comparison of VLDLR mRNA expression in Lueyang (LY) chickens with or without ATAATA(A/C)T and in Nick Chick (NC) chickens. LY without the ATAATA(A/C)T group was used as a calibrator. The expression levels of VLDLR mRNA in LY with ATAATA(A/C)T and NC are shown as the fold-change relative to the calibrator. The columns represent the mean $\pm \mathrm{SD}$ of the fold change for LY with ATAATA(A/C)T ( $\mathrm{n}=6$ ), LY without $\operatorname{ATAATA}(\mathrm{A} / \mathrm{C}) \mathrm{T}(\mathrm{n}=6)$ and $\mathrm{NC}(\mathrm{n}=6)$ in liver and ovary. There was no significant difference $(\mathrm{p}>0.05)$ in VLDLR mRNA expression among the groups within a given organ (liver and ovary). Statistical significance was test by ANOVA followed by Duncan's multiple range test. 


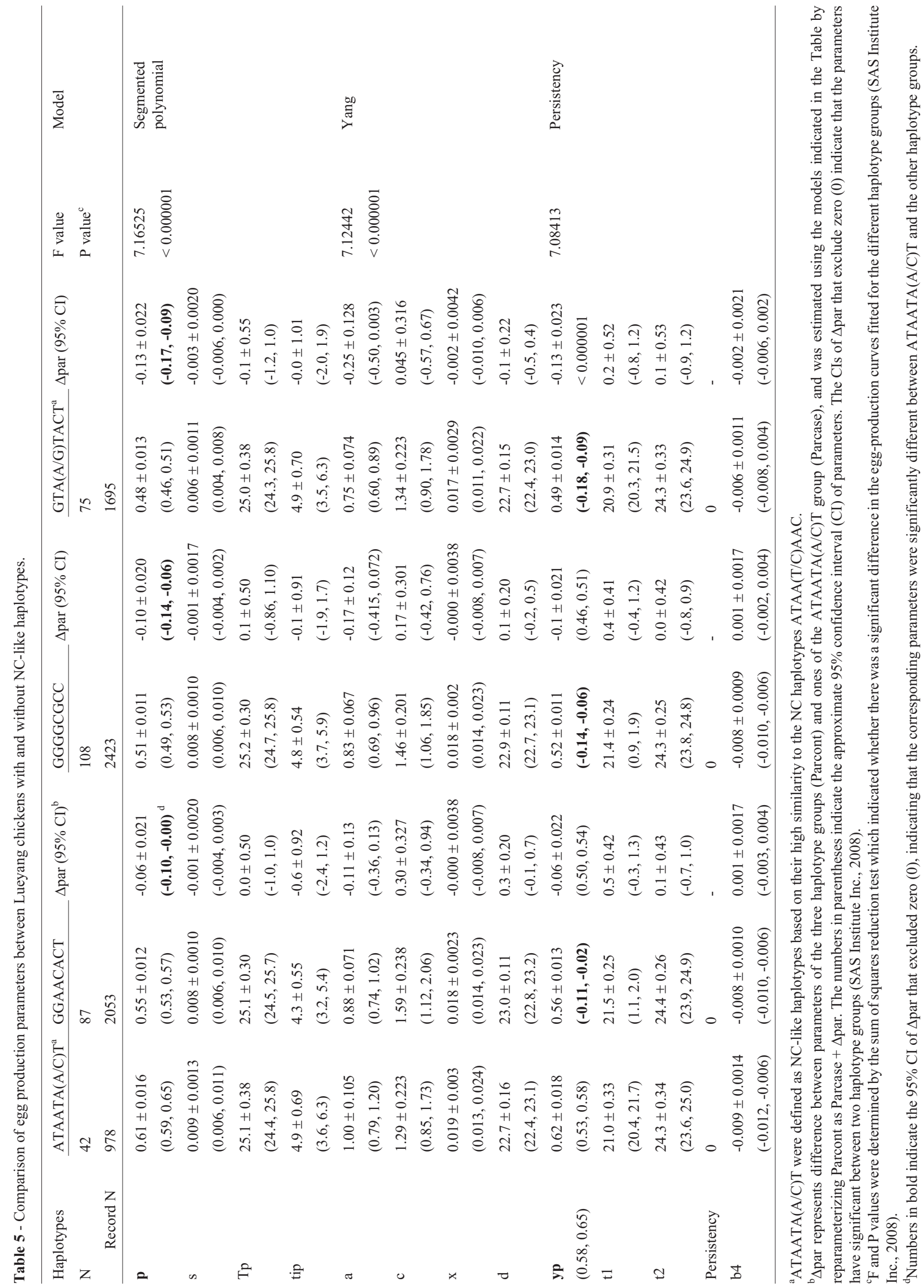


of their daughters (Elkin and Zhong, 2002). To examine the distribution of the RO mutation in LY and assess whether it is a major factor resulting in the low egg production of LY, $50 \mathrm{LY}$ hens and 30 cocks were screened for the mutation. All individuals showed two bands ( $85 \mathrm{bp}$ and $474 \mathrm{bp}$ ), indicating that the mutation did not exist in the samples that were tested (Figure 4).

\section{Discussion}

We have previously observed two important characteristics of LY chickens, namely, their low egg production and large intraspecies variability. In this study, all birds from the same hatch were reared in the same environment. This experimental design allowed us to conclude that these features were mainly determined by genetic factors. Large intraspecies variability implies that some variants that regulate egg production may be separable in the LY breed, which, thus, could provide an interesting animal model for identification of the relevant mutations.

Although VLDLR is a promising candidate gene for modulating egg production. our knowledge of the relationship between VLDLR variants and egg production is still incomplete; the only mutation known to adversely affect hen reproduction is the RO mutation (Bujo et al., 1995). No studies have specifically sought to map the distribution of VLDLR variants among different breeds such that we do not know whether there are some favorable, specificallyselected variants in the VLDLR. In this study, the genome sequence covering the whole VLDLR and a $5 \mathrm{~kb}$ region upstream was re-sequenced and eight SNPs accounting for $87 \%$ of all variants were genotyped in two commercial egg-laying breeds ( $\mathrm{NC}$ and LohB) and LY. The data obtained from this sequencing showed that: (1) VLDLR variants were almost fixed in the commercial breeds, whereas there was considerable haplotype diversity in LY (Table 4) and (2) two LY haplotypes, similar to NC haplotypes, were significantly associated with high egg production (Table 5).

A decrease in genetic variation can be caused by selection, inbreeding and genetic drift. Clarification of the roles of these factors in shaping the distribution patterns of VLDLR variants among these breeds is key to understanding the influence of VLDLR variants on egg production. For breeders, inbreeding is a useful way of fixing traits within a short period of time and has been widely used to breed pure lines. However, the effect of inbreeding on genome variants is general instead of being confined to a specific gene (Charlesworth, 2003). Genetic drift can also change the allele frequency, leading to the fixation or disappearance of variants through random sampling (Masel, 2011). As shown here, four haplotypes were found in NC and LohB (Table 4). These haplotypes showed high similarity between the two breeds, with the first four bases being completely conserved and NChap2 occurring in LohB at a particularly high frequency of 0.925 (Table 4). NC is a popular white-egg laying breed, initially bred by H\&N International based on the Kimber Leghorn in 1945. LohB was bred from New Hampshire and other breeds that lay brown eggs. The different genetic backgrounds of these breeds suggests that the possibility of highly similar haplotypic profiles appearing in different breeds should be small if inbreeding and genetic drift are the determining factors.

The effect of selection on genetic variants within the genome is immense. Human-driven selection not only increases the frequency of favorable variants in the population, but also leads to a reduction or loss of nucleotide diversity at some linked neutral loci, a phenomenon often referred to as "genetic hitchhiking" or "selective sweep" (Elferink et al., 2012). Sundström et al. (2004) detected Z chromosome variability in 13 introns of nine genes, including VLDLR, and confirmed that there was a signature of selection on chromosome $\mathrm{Z}$. If the decrease in haplotype diversity in $\mathrm{NC}$ and LohB were caused by selection, we would expect these haplotypes to represent functional haplotypes associated with high egg production. Fortunately, considerable haplotypic diversity was maintained in LY, which provides an opportunity to analyze the phenotypic effect of these haplotypes. As shown here, two NC-like haplotypes were significantly associated with high peak production (Table 5, Figure 2), a finding compatible with "selective sweep" having a key role in reducing VLDLR variations.

Han et al. (2009) suggested that the level of VLDLR mRNA expression in ovary was a determining factor in the reproductive phenotype. However, our results showed that the expression level of this receptor was not higher in the $\mathrm{NC}$ or NC-like haplotypes than in the other haplotypes (Figure 3), which indicated that high egg production was

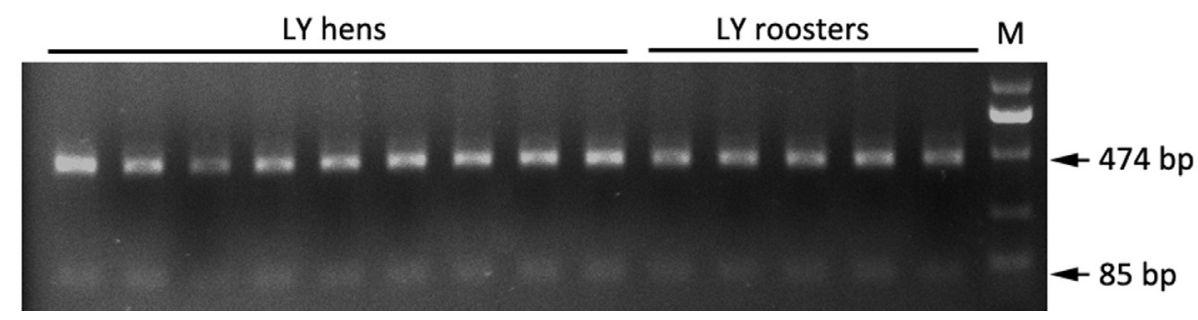

Figure 4 - Detection of the RO mutation in LY hens $(n=50)$ and roosters $(n=30)$. The RO mutation was detected using PCR-RFLP. Wild-type individuals had two bands ( $85 \mathrm{bp}$ and $474 \mathrm{bp}$ ) and those with the RO mutation had four bands ( $85 \mathrm{bp}, 108 \mathrm{bp}, 451 \mathrm{bp}$ and $474 \mathrm{bp}$ ). 
not necessarily associated with enhanced VLDLR expression. On the other hand, variations in mRNA expression do not necessarily couple with changes in protein activity and function (Nikinmaa and Waser, 2007). After being synthesized, VLDLR needs to be transferred to the oocyte periphery to function (Bujo et al., 1994). Fluorescence in situ hybridization and ligand-binding assays will be needed to clarify whether the two phenotype-associated haplotypes are correlated with changes at the protein level.

In summary, our data indicate that VLDLR variants were almost fixed in selectively bred NC and LohB chickens, whereas haplotypic diversity was maintained in LY chickens. The finding that different egg-laying breeds shared similar haplotypic profiles and that the haplotypes exerted a phenotype effect suggested that selection may be a key factor in shaping the current distribution patterns of VLDLR variants. To our knowledge, this is the first report to specifically focus on the distribution of VLDLR variants in commercial and indigenous breeds of chicken. The results provide new support for VLDLR as a promising candidate gene for modulating egg production.

\section{Acknowledgments}

This research was supported by the Fundamental Research Funds for the Central Universities (grant no. 2014YB004), the Natural Science Basic Research Plan in Shaanxi Province of China (grant no. 2014JQ3106), the National Natural Science Foundation (grant no. 31401051) and China Agriculture Research Systems (grant no. CARS-41-S23).

\section{References}

Atzmon G, Blum S, Feldman M, Lavi U and Hillel J (2007) Detection of agriculturally important QTLs in chickens and analysis of the factors affecting genotyping strategy. Cytogenet Genome Res 117:327-337.

Bujo H, Hermann M, Kaderli MO, Jacobsen L, Sugawara S, Nimpf J, Yamamoto T and Schneider WJ (1994) Chicken oocyte growth is mediated by an eight ligand binding repeat member of the LDL receptor family. EMBO J 13:5165-5175.

Bujo H, Yamamoto T, Hayashi K, Hermann M, Nimpf J and Schneider WJ (1995) Mutant oocytic low density lipoprotein receptor gene family member causes atherosclerosis and female sterility. Proc Natl Acad Sci USA 92:9905-9909.

Bujo H, Hermann M, Lindstedt KA, Nimpf J and Schneider WJ (1997) Low density lipoprotein receptor gene family members mediate yolk deposition. J Nutr 127:801S-804S.

Cao DG, Zhou Y, Lei QX, Han HX, Li FW, Li GM, Wu B and Wang ZJ (2012) Associations of very low density lipoprotein receptor (VLDLR) gene polymorphisms with reproductive traits in a Chinese indigenous chicken breed. J Anim Vet Adv 11:3662-3667.

Charlesworth D (2003) Effects of inbreeding on the genetic diversity of populations. Philos Trans R Soc Lond B Biol Sci 358:1051-1070.
Elferink MG, Megens HJ, Vereijken A, Hu X, Crooijmans RP and Groenen MA (2012) Signatures of selection in the genomes of commercial and non-commercial chicken breeds. PLoS One 7:e32720.

Elkin RG and Zhong Y (2002) Assessment of reproductive function in mutant restricted ovulator carrier roosters. Poult Sci 81:1280-1282.

Fialho FB and Ledur MC (1997) Segmented polynomial model for estimation of egg production curves in laying hens. $\mathrm{Br}$ Poult Sci 38:66-73.

Gholami M, Reimer C, Erbe M, Preisinger R, Weigend A, Weigend S, Servin B and Simianer H (2015) Genome scan for selection in structured layer chicken populations exploiting linkage disequilibrium information. PLoS One 10:e130497.

Goto T, Ishikawa A, Goto N, Nishibori M, Umino T and Tsudzuki M (2014) Mapping of main-effect and epistatic quantitative trait loci for internal egg traits in an $\mathrm{F}_{2}$ resource population of chickens. J Poult Sci 51:375-386.

Grossman M, Grossman TN and Koops WJ (2000) A model for persistency of egg production. Poult Sci 79:1715-1724.

Han D, Haunerland NH and Williams TD (2009) Variation in yolk precursor receptor mRNA expression is a key determinant of reproductive phenotype in the zebra finch (Taeniopygia guttata). J Exp Biol 212:1277-1283.

Honkatukia M, Tuiskula-Haavisto M, Arango J, Tabell J, Schmutz M, Preisinger R and Vilkki J (2013) QTL mapping of egg albumen quality in egg layers. Genet Sel Evol 45:31.

Livak KJ and Schmittgen TD (2001) Analysis of relative gene expression data using real-time quantitative PCR and the ${ }^{-} \triangle \Delta$ CT method. Methods 25:402-408.

Liu W, Li D, Liu J, Chen S, Qu L, Zheng JX, Xu GY and Yang N (2011) A genome-wide SNP scan reveals novel loci for egg production and quality traits in white leghorn and brown-egg dwarf layers. PLoS One 6:e28600.

Masel J (2011) Genetic drift. Curr Biol 21:R837-R838.

Meng XT, Hou NN, Wang XJ, Jiao HC, Zhao JP, Song ZG and Lin H (2013) Increased hepatic yolk precursor synthesis, secretion and facilitated uptake by follicles are involved in the rejuvenation of reproductive performance of molted hens (Gallus gallus domesticus). Gen Comp Endocrinol 194:198-207.

Nikinmaa M and Waser W (2007) Molecular and cellular studies in evolutionary physiology of natural vertebrate populations: Influences of individual variation and genetic components on sampling and measurements. J Exp Biol 210:18471857.

Rubin CJ, Zody MC, Eriksson J, Meadows JR, Sherwood E, Webster MT, Jiang L, Ingman M, Sharpe T, Ka S, et al. (2010) Whole-genome resequencing reveals loci under selection during chicken domestication. Nature 464:587-591.

Sambrook J and Russell DW (2006) Purification of nucleic acids by extraction with phenol: Chloroform. CSH Protocols, Cold Spring Harbor, NY, pii:prot4455.

SAS Institute Inc. (2008) SAS/STAT 9.2 User's Guide. SAS Institute Inc., Cary, NC.

Sasaki O, Odawara S, Takahashi H, Nirasawa K, Oyamada Y, Yamamoto R, Ishii K, Nagamine Y, Takeda H, Kobayashi $\mathrm{E}$, et al. (2004) Genetic mapping of quantitative trait loci affecting body weight, egg character and egg production in $\mathrm{F}_{2}$ intercross chickens. Anim Genet 35:188-194. 
Savegnago RP, Cruz VA, Ramos SB, Caetano SL, Schmidt GS, Ledur MC, El Faro L and Munari DP (2012) Egg production curve fitting using nonlinear models for selected and nonselected lines of White Leghorn hens. Poult Sci 91:29772987.

Sun C, Lu J, Yi G, Yuan J, Duan Z, Qu L, Xu G, Wang K and Yang N (2015a) Promising loci and genes for yolk and ovary weight in chickens revealed by a genome-wide association study. PLoS One 10:e137145.

Sun C, Qu L, Yi G, Yuan J, Duan Z, Shen M, Qu L, Xu G, Wang K and Yang N (2015b) Genome-wide association study revealed a promising region and candidate genes for eggshell quality in an F2 resource population. BMC Genomics 16:565.

Sundström H, Webster MT and Ellegren H (2004) Reduced variation on the chicken $\mathrm{Z}$ chromosome. Genetics 167:377-385.

Tuiskula-Haavisto M, Honkatukia M, Vilkki J, de Koning DJ, Schulman NF and Mäki-Tanila A (2002) Mapping of quantitative trait loci affecting quality and production traits in egg layers. Poult Sci 81:919-927.

Wang C, Li SJ, Yu WH, Xin QW, Li C, Feng YP, Peng XL and Gong YZ (2011) Cloning and expression profiling of the VLDLR gene associated with egg performance in duck (Anas platyrhynchos). Genet Sel Evol 43:29.

Wolc A, Arango J, Jankowski T, Dunn I, Settar P, Fulton JE, O'Sullivan NP, Preisinger R, Fernando RL, Garrick DJ, et al. (2014) Genome-wide association study for egg production and quality in layer chickens. J Anim Breed Genet 131:173-182.

Wolc A, Arango J, Settar P, Fulton JE, O'Sullivan NP, Preisinger R, Habier D, Fernando F, Garrick DJ, Hill WG, et al. (2012) Genome-wide association analysis and genetic architecture of egg weight and egg uniformity in layer chickens. Anim Genet 43:87-96.

Wu Y, Pi JS, Pan AL, Du JP, Shen J, Pu YJ and Liang ZH (2015) Two novel linkage SNPs of VLDLR gene intron 11 are associated with laying traits in two quail populations. Arch Anim Breed 58:1-6.

Xu H, Zeng H, Luo C, Zhang D, Wang Q, Sun L, Yang L, Zhou M, Nie Q and Zhang X (2011) Genetic effects of polymorphisms in candidate genes and the QTL region on chicken age at first egg. BMC Genet 12:e33.

Yang N, Wu C and McMillan I (1989) New mathematical model of poultry egg production. Poult Sci 68:476-481.

Yi G, Shen M, Yuan J, Sun C, Duan Z, Qu L, Dou T, Ma M, Lu J, Guo J, et al. (2015) Genome-wide association study dissects genetic architecture underlying longitudinal egg weights in chickens. BMC Genomics 16:e746.

Yuan J, Sun C, Dou T, Yi G, Qu L, Qu L, Wang K and Yang N (2015) Identification of promising mutants associated with egg production traits revealed by genome-wide association study. PLoS One 10:e140615.

\section{Supplementary material}

The following online material is available for this article:

Table S1 - Primer sets used for PCR and DNA sequencing of the VLDLR gene.

Table S2 - Information for all SNPs found in the VLDLR

Associate Editor: Dario Grattapaglia

License information: This is an open-access article distributed under the terms of the Creative Commons Attribution License (type CC-BY), which permits unrestricted use, distribution and reproduction in any medium, provided the original article is properly cited. 